\title{
Non-smooth Feedback Control for a Class of Constraint Systems
}

\author{
Chen Weijun $^{*}$ and Jia Weifeng
}

Anyang Normal University, Anyang, Henan 455000, China

\begin{abstract}
Beginning from the second-order input constraint systems, the paper pays attention to study the control problem for a class of the constraint systems with the nalyzes the stability of the second-order input constraint systems under the condition of linear state feedback. On the basis of that, it gives the non-smooth feedback control law of the third-order state constraint systems. At the same time, it sets up the relationship between system's steady state and its initial conditions. Finally, it has verified the validity of the conclusion by simulation.
\end{abstract}

Keywords: Limited constraint, non-smooth control, saturation nonlinearity.

\section{INTRODUCTION}

In the daily life, systems usually work under the condition of some constraints or limits which is often showed by one link from systems that has been affected by a certain degree of the characteristics of saturation nonlinearity. Influenced by saturation nonlinearity, this kind of systems will make the analysis and design of the systems difficult because it is not only deferent from normal one in system performance, but also difficult to adopt common means to judge its stability, especially for the situation that the change of some system state affected by the saturation nonlinearity, under which it is very difficult to use existing methods to analyze the stability of the systems. Therefore, judging from the researches on saturation nonlinearity control problem, there is not an effective theoretical framework to solve the stability of the systems and the design. However, although saturation nonlinearity on independent variable is continuous, it is not continuous for the derivative of variable. So that is a kind of non- smooth nonlinearity which has the system with saturation nonlinear. What's more, in essence, it is a class of nonsmooth system, whose control problem can be considered from non-smooth control theories and design methods. Just from the perspective of non-smooth feedback design view, this paper studies that the change of system state has the control problem of saturation non-linear constraint systems.

In fact, as for the control problem of the system with saturation nonlinearity, there has been a lot of research achievements published. Reference [1] gives the sufficient and necessary condition of system stability for the reliability problem of a class of linear saturation nonlinear system. That condition shows that, in the case that the state of linearity system is completely affected by saturation nonlinearity, the system would make the global asymptotic stability come true if the system matrix met certain conditions. Especially for the second-order system, it is required that System Matrix A belongs to Hurwitz's with a line which has diagonally domi- nant characteristic. Reference [2], based on Reference [1], further discusses the convergence problem for the secondorder system. In regard to the study of the second-order system, it can be found that Reference [3] studies detailed the track of a class of the second-order system under the condition of saturation constraints [4]. And input limited system is discussed in Reference [4] where there are the design methods that linear system gets the maximum convergence rate of elliptic invariant sets in the sate of saturation constraints. These research results generally focus on a class of systems whose saturation nonlinearity has an effect on all the system states, and the restricted states of many systems are usually limited in practice [5-7]. Thus, the conclusion above can not be used directly.

This paper studies a class of systems which is in the limited state. The systems have three states, among which there is a kind of state-saturation nonlinearity-which is often seen under the condition of actuator torque limited. Firstly, the paper starts with the feedback designs with the state of the second-order input constraint systems and draws the conclusion of the stability of the systems that are in the status of linearity. And then, the linear state feedback controller gotten by the second-order system directly extends to the target system and gets non-smooth feedback controller. At the same time, it further discusses the relationship between system stability and initial conditions and establishes the relationship between the two. Finally, it has verified the conclusion under different initial conditions by numerical simulation.

\section{THE PROBLEM DESCRIPTION}

Limited systems may have a characteristic of saturation which is often showed by the control input of systems and the actuator output of ones. This paper investigates a class of the systems which are in constrained states. There are the following forms:

$$
\left\{\begin{array}{c}
\dot{x}_{1}=x_{2} \\
\dot{x}_{2}=\operatorname{sat}\left(x_{3}\right) \\
\dot{x}_{3}=u
\end{array}\right.
$$


$\mathrm{x}_{1}, \mathrm{x}_{2}$ and $\mathrm{x}_{3}$ are system states. $\mathrm{u}$ refers to the control input of systems. Saturation function $\operatorname{sat}(u)$ is defined as:

$\operatorname{sat}(u)=\left\{\begin{array}{c}u, u \leq 1 \\ \operatorname{sgn}(u)>1\end{array}\right.$

If it is assumed that system state and initial value have been known, a state feedback controller $\mathrm{u}$ is designed, this makes the state of System 1 convergent.

\section{THE NON-SMOOTH FEEDBACK DESIGN}

\subsection{The State Feedback Design of Second-order Con- strained System}

To solve the problem of the control law design of the system (1), we may consider first the second-order input constrained system which has the following forms:

$\left\{\begin{array}{c}\dot{x}_{1}=x_{2} \\ \dot{x}_{2}=\operatorname{sat}\left(u_{0}\right)\end{array}\right.$

In order to design for the original system, we take $u$ as linear feedback, that is, $u_{0}=\left(-k_{1} x_{1}-k_{2} x_{2}\right)$. Then, the system (3) can be converted into:

$\left\{\begin{array}{c}\dot{X}_{1}=x_{2} \\ \dot{X}_{2}=\operatorname{sat}\left(-k_{1} X_{1}-k_{2 X}\right)\end{array}\right.$

Among them, $\mathrm{k}_{1}>0, \mathrm{k}_{2}>0$. Obviously, the equilibrium point of the system is still $\left(x_{1}, x_{2}\right)=(0,0)$, but there is no one in the following:

domain- $\left\{\left(x_{1}, X_{2}\right)||-k_{1} X_{1}-k_{2} X_{2} \mid>1\right\}$.

If the system (4) is stable, according to the linear feedback, we can make a further design for the original system. Below we discuss the salability of the system (4).

For the convenience of analysis, we transform the system (4) into the following coordinates:

$\left\{\begin{array}{c}Z_{1}=-k_{1} X 1-k_{2} X 2 \\ Z_{2}=X_{2}\end{array}\right.$

Then the system (4) is turned into:

$\left\{\begin{array}{c}\dot{z}_{1}=-k_{2} \operatorname{sat}\left(z_{1}\right)-k_{1 z_{2}} \\ \dot{z}_{2}=-\operatorname{sat}\left(z_{1}\right)\end{array}\right.$

To judge the stability of the system (6), we take the following positive definite function:

$v_{1}=a_{1} \int_{0}^{z_{l}} \operatorname{sat}(t) d t+\frac{1}{2} a_{2} z_{2}^{2}$
Among them, $a_{1}>0, a_{2}>0$. The time derivative of $v_{1}$, along the track of the system (6), is:

$V_{1}=-\operatorname{arsat}_{1}\left(Z_{1}\right)\left[k_{2} \operatorname{sat}\left(Z_{1}\right)+k_{12}\right]+\operatorname{a}_{2} Z_{2} \operatorname{sat}\left(Z_{1}\right)$

We let $\mathrm{a}_{2}=\mathrm{a}_{1} \mathrm{k}_{1}$, have:

$\dot{V}_{1}=-a_{1} k_{2} \operatorname{sat}^{2}\left(z_{1}\right) \leq 0$

Based on the invariant set principle, the track of the system (6) when it is in $t \rightarrow \infty$ will converge to the invariant set of $\left\{\left(Z_{1}, Z_{2}\right) \mid Z_{1}=0\right\}$. While $Z_{1} \rightarrow 0, \dot{Z}_{1} \rightarrow 0$. Thus, $Z_{2} \rightarrow 0$ and the system (6) is asymptotically stable. According to the coordinate transformation (5), as $t \rightarrow \infty$, $X_{1} \rightarrow 0$ and $X_{2} \rightarrow 0$. So the system (4) is asymptotically stable. That conclusion can be described by the lemma as follows:

Lemma 1: As for the system (3), the second-order input constrained system, influenced by the following linear feedback control law;

$u_{0}=-k_{1} x_{1}-k_{2} x_{2}\left(k_{1}>0, k_{2}>0\right)$

the closed-loop system is globally asymptotically stable.

\subsection{The Non-smooth Feedback Design}

For the original system (1), if the state variable $\mathrm{x}_{3}$ can reproduce completely the linear feedback control law (10), the closed loop system will be asymptotically stable according to Lemma 1. So we can take:

$X_{3}=-k_{1 X 1}-k_{2} X_{2}$

And we get:

$\dot{X}_{3}=-k_{1} \dot{X}_{1}-k_{2} \dot{X}_{2}=-k_{1} X_{2}-k_{2} \operatorname{sat}\left(x_{3}\right)$

Then, the control law $u=-k_{1} x_{2}-k_{2} \operatorname{sat}\left(x_{3}\right)$, which is obviously a non-smooth function of the state variable. Under the action of the control law, the closed-loop system is:

$\left\{\begin{array}{c}\dot{X}_{1}=x_{2} \\ \dot{X}_{2}=\operatorname{sat}\left(x_{3}\right) \\ \dot{X}_{3}=-k_{1} x_{2}-k_{2} \operatorname{sat}\left(x_{3}\right)\end{array}\right.$

However, the formula (11) is gotten by the integral for the state variable $x_{3}$. Therefore, the gotten results are different under different initial conditions. The following discussion is about the stability of the closed-loop system (13) under different initial conditions. We take $x_{l}(0)=x_{3_{0}}$, $x_{2}(0)=x_{3_{0}}, x_{3}(0)=x_{3_{0}}$ as the initial conditions of the system (1). Then the equation (12) has:

$\int_{0}^{t} x_{3} d t=\int_{0}^{t}\left[-k_{1} X_{2}-k_{2} \operatorname{Sat}\left(x_{3}\right)\right] d t$

We thereby get: 
$x_{3}(t)-x_{3}=-k_{1}\left[x_{1}(t)-x_{1}\right]-k_{2}\left[x_{2}(t)-x_{2}\right]$

That is to say:

$x_{3}(t)=-k_{1} x_{1}-k_{2} x_{2}+d_{0}$

Among them, $\boldsymbol{\delta}_{0}=X_{3}+k_{1} x_{0}+k_{2} X_{2}{ }_{0}$. Since the system state $\mathrm{x} 3$ has been known, the system (13) can convert into the following the second-order constrained system:

$$
\left\{\begin{array}{c}
\dot{x}_{1}=x_{2} \\
\dot{x}_{2}=\operatorname{sat}\left(-k_{1} x_{1}-k_{2} x_{2}+\delta_{0}\right)
\end{array}\right.
$$

And the system (17) is transformed into the following coordinates:

$\left\{\begin{array}{c}\left.\tilde{z}_{1}=-k_{1} x_{1}-k_{2} x_{2}+d_{0}\right) \\ \tilde{z}_{2}=x_{2}\end{array}\right.$

Then it can be turned into:

$\left\{\begin{array}{c}\dot{\tilde{z}}_{1}=-k_{2} \operatorname{sat}\left(\tilde{z}_{1}\right)-k_{1} \tilde{z}_{2} \\ \dot{\tilde{z}}_{2}=-\operatorname{sat}\left(\tilde{z}_{1}\right)\end{array}\right.$

Both the system (19) and the system (6) are consistent in form except that their definitions about state variable are different. So positive definite function is still used:

$v_{2}=a 1 \int_{0}^{\tilde{z}_{1}} \operatorname{sat}(t) d t+\frac{1}{2} a_{2} \tilde{z}_{2}^{2}$

Then the system (19) gotten is asymptotically stable, which shows that, at $t \rightarrow \infty,-k_{1} X_{1}-k_{2} X_{2}+\delta_{0} \rightarrow 0$ and $X_{2} \rightarrow \infty$. Thus, $X_{1} \rightarrow \delta_{0} / k_{1}$. And then we may draw the conclusion below by the closed-loop system (13).

Theorem 1: Under the action of the following control law of non-smooth feedback:

$\tilde{u}=-k_{1} x_{2}-k_{2} \operatorname{sat}\left(x_{3}\right)\left(\mathrm{k}_{1}>0, \mathrm{k}_{2}>0\right)$

the third-order constrained system (1) when it is in $t \rightarrow \infty$, the system sate is $X_{1} \rightarrow \delta_{0} / k_{1}, X_{2} \rightarrow 0, X_{3} \rightarrow 0$.

Theorem 1 shows that the non-smooth feedback control law (21) may make the state of the third-order constrained system (1) bounded stable. The feedback control law is only connected with $\mathrm{x}_{2}$ and $\mathrm{x}_{3}$, not the system state $\mathrm{x}_{1}$. The control law contains the limited output of the system state $x_{3}$, is the non-smooth function of the system state $x_{3}$ and to a certain extent it can limit the amount of feedback control.

In addition, under the action of the non-smooth feedback control law (21), the third-order constrained system (1) is that its state is bounded stable. That is to say, its states converge to 0 except that the state $\mathrm{x}_{1}$ eventually converges to a constant value $\delta_{0} / k_{1}$, which is influenced by the form of the feedback control law (21). Because of that the feedback control does not contain the system state $\mathrm{x}_{1}$, eventually the state of the system $\mathrm{x}_{1}$ does not converge to 0 . The final value of $\mathrm{x}_{1}$ will reflect the steady-state error of the system, which is distinctly related to the initial condition $\delta_{0}$ and the control parameter $\mathrm{k}_{1}$. When the initial condition is zero, i.e., the initial condition of the original system (1) satisfies:

$X_{3}+k_{1 X 1_{0}}+k_{2 X 2_{0}}=0$

the system will converge to the origin.

\section{THE SIMULATION STUDY}

We take $\mathrm{k}_{1}=2, \mathrm{k}_{2}=1$, and then, respectively under different initial conditions, conduct the simulation study on the closed-loop systems which is under the action of the control law (21). Fig. (1) shows that when the initial condition is $\left(x_{1}, x_{2}, x_{3}\right)=(1,2,3)$, the response time curves (see Fig. 1a) and the controller output (see Fig. 1b) in the closedloop system state. According to Fig. (1a), we know that both the sate of $x_{2}$ and the state of $x_{3}$ converge to the origin at this moment, but the state of $\mathrm{x}_{1}$ converges to 3.5 , not to the origin. Then we get $\delta_{0}=3+2^{\prime} 1+1^{\prime} 2=7$. Based on Theorem 1, the state of $\mathrm{x}_{1}$ should con verge to $\delta_{0} / \mathrm{k}_{1}=3.5$, which is consistent with the results of the simulation. Fig. (2) displays that when the initial condition is $\left(x_{0}, X_{2}, X_{3}\right)=(1,2,-4)$, the response time curves (see Fig. 2a) and the controller output (see Fig. 2b) in the closed-

loop system state. If the initial condition is $\left(X_{1}, X_{2} n_{n}\right.$, $\left.X_{3_{0}}\right)=(1,2,-4)$, the equation $(22), \delta_{0}=-4+2^{\prime} 1+1^{\prime} 2$ $=0$, is true. According to Theorem 1, we may know that the stats of $x_{1}, x_{2}$ and $x_{3}$ will converge to the origin. Seen from the controller outputs under different initial conditions, controller outputs have an obvious characteristic of saturation, but the non-smooth feature does not affect its control effect.

\section{CONCLUSION}

This paper designs the linear state feedback control laws for the second-order input constrained systems so as to guarantee the asymptotic stability of the closed-loop systems. And then it generalizes the conclusion to the control law design problem of a class of the third-order state constrained systems and gives a kind of non-smooth state feedback control law. Based on that, the paper discusses the relationship between the system steady states and the initial conditions of system states and makes the quantitative relations between the two clear, which can be considered in the design. Of course, the non-smooth state feedback control law gotten can not guarantee the asymptotic stability of the closed-loop systems for the origin. That point still should be realized by choosing proper initial conditions. 


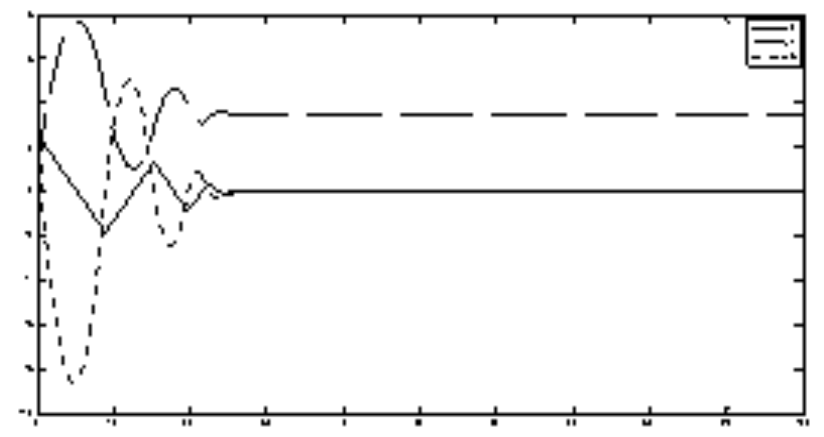

(a) The response time curves of the closed-loop systems

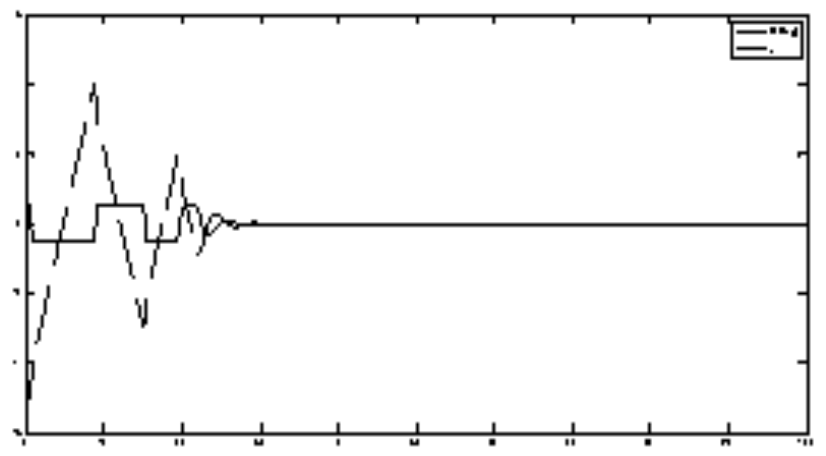

(b) The outputs of the controller and saturation

Fig. (1). The simulation results with the initial conditions $(1,2,3)$.

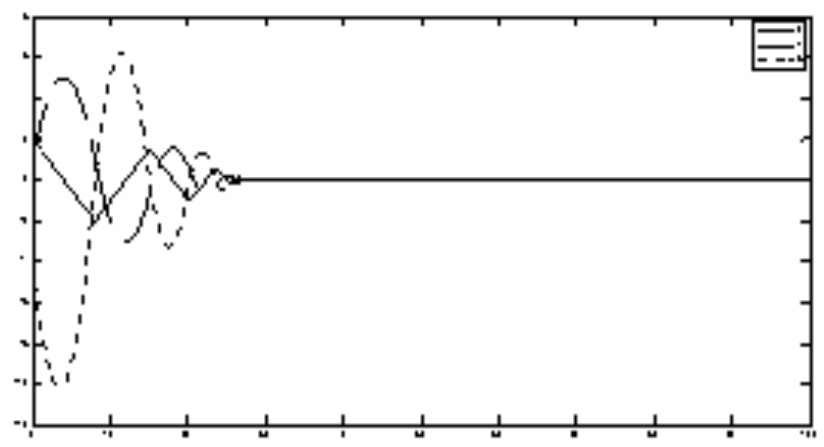

(a) The response time curves of the closed-loop systems

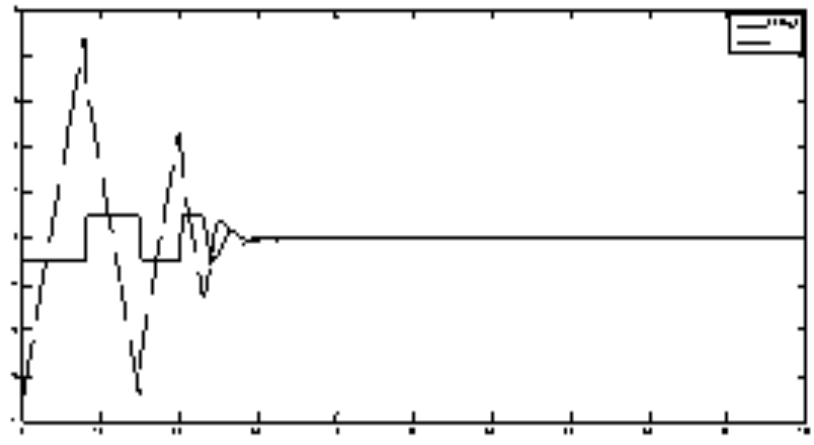

(b) The outputs of the controller and saturation

Fig. (2). The simulation results with the initial conditions $(1,2,-4)$. 


\section{CONFLICT OF INTEREST}

We declare that we have no financial and personal relationships with other people or organizations that can inappropriately influence our work, there is no professional or other personal interest of any nature or kind in any product, service and/or company that could be construed as influencing the position presented in, or the review of, the manuscript entitled, "Non-smooth Feedback Control for a Class of Constraint Systems".

\section{ACKNOWLEDGEMENTS}

On the completion of my thesis, I should like to express my deepest gratitude to all those kindness and advices have made this work possible. I am greatly indebted to my friend $\mathrm{Gu}$ Jinbai who gave me valuable instructions and has improved me in language. His effective advice, shrewd comments have kept the thesis in the right direction. I would like to thank my partner Jia Weifeng for their friendship and constructive suggestions, he constantly encouraged me when I felt frustrated with this dissertation.

\section{REFERENCES}

[1] F. Albertini, and D. D'Alessandro, "Asymptotic stability of continuous-time systems with saturation nonlinearities," Systems \& Control Letters, vol. 29, pp. 175-180, 1996.

[2] F. Albertini, D. D'Alessandro, and A.D.B. Paice, "Further conditions on the stability of continuous time systems with saturation," IEEE Transactions on Circuits and Systems-I: Fundamental Theory and Applications, vol. 47, no. 5, pp. 723-729, 2000.

[3] T. Hu, and Z. Lin, "A complete stability analysis of planar linear systems under saturation," IEEE Transactions on Circuits and Systems-I: Fundamental Theory and Applications, vol. 47, no. 4, pp. 498-512, 2000.

[4] T. Hu, Z. Lin, and Y. Shamash, "On maximizing the convergence rate for linear systems with input saturation," IEEE Transactions on Automatic Control, vol. 48, no. 7, pp. 1249-1253, 2003.

[5] J.M. Gonçalves, "L2-gain of double integrators with saturation nonlinearity," IEEE Transactions on Automatic Control, vol. 47 , no. 12 , pp. 2063-2068, 2002.

[6] C. -Y. Kao, "On the L2-gain of a double integrator with feedback loop saturation nonlinearity," IEEE Transactions on Automatic Control, vol. 46, no. 3, pp. 501-504, 2001.

[7] G. Grimm, J. Hatfield, I. Postlethwaite, A.R. Teel, M.C. Turner, and L. Zaccarian, "Antiwindup for stable linear systems with input saturation: An LMI-based synthesis," IEEE Transactions on Automatic Control, vol. 48, no. 9, pp. 1509-1525, 2003.

(C) Weijun and Weifeng; Licensee Bentham Open.

This is an open access article licensed under the terms of the Creative Commons Attribution Non-Commercial License (http://creativecommons.org/licenses/by-nc/3.0/) which permits unrestricted, non-commercial use, distribution and reproduction in any medium, provided the work is properly cited. 